\title{
Dynamics of a discrete-time predator-prey system
}

\author{
Ming Zhao', Zuxing Xuan²* and Cuiping $\mathrm{Li}^{1}$
}

"Correspondence:

zuxingxuan@163.com

${ }^{2}$ Beijing Key Laboratory of

Information Service Engineering,

Department of General Education,

Beijing Union University, Beijing,

100101, China

Full list of author information is

available at the end of the article (c) 2016 Zhao et al. This article is distributed under the terms of the Creative Commons Attribution 4.0 International License (http://creativecommons.org/licenses/by/4.0/), which permits unrestricted use, distribution, and reproduction in any medium, provided you give appropriate credit to the original author(s) and the source, provide a link to the Creative Commons license, and indicate if changes were made.

\section{Introduction}

It is well known that the Lotka-Volterra predator-prey model $[1,2]$ is one of the most important population models. There are also many other predator-prey models of various types that have been extensively investigated, and some of the relevant work may be found in [3-8]. These researches dealing with specific interactions have mainly focused on continuous predator-prey models with two variables. However, discrete-time models described are more reasonable than the continuous-time models when populations have nonoverlapping generations. Moreover, using discrete-time models is more efficient for computation and numerical simulations [9]. For example, in [10], the authors use the forward Euler discrete scheme to obtain a discrete-time predator-prey system and prove that the system undergoes flip bifurcation and Neimark-Sacker bifurcation. Recently, the complex dynamics of a discrete-time predator-prey system is investigated in [11]. By analysis it is proved that the discrete-time model has different properties and structures compared with the continuous one. Such systems discussed as discrete-time models can also be found in [12-21] and references therein.

In this paper, we consider the following discrete-time predator-prey system:

$$
\left\{\begin{array}{l}
x_{n+1}=a x_{n}\left(1-x_{n}\right)-b x_{n} y_{n}, \\
y_{n+1}=-c y_{n}+d x_{n} y_{n},
\end{array}\right.
$$


where $x$ and $y$ represent population densities of a prey and a predator, respectively, and $a, b, c, d$ are positive parameters. Here $a$ represents the natural growth rate of the prey in the absence of predators, $b$ represents the effect of predation on the prey, $c$ represents the natural death rate of the predator in the absence of prey, and $d$ represents the efficiency and propagation rate of the predator in the presence of prey. In [22], the authors investigated the discrete-time predator-prey system for $c=0$, and they proved that there are flip and Hopf bifurcations and there exists a chaotic phenomenon in the sense of Marotto. In this paper, we study system (1) for $c \neq 0$.

Motivation of this paper is to investigate system (1) in detail. Here we derive the conditions of existence for flip bifurcation and Neimark-Sacker bifurcation by using bifurcation theory and the center manifold theorem $[23,24]$. Numerical simulations are given to support the theoretical results and display new and interesting dynamical behaviors of the system. More specifically, this paper presents the period-6, -11, -16, -18, -20, -21, -24, -27, -37 orbits, attracting invariant cycles, quasi-periodic orbits, nice chaotic behaviors, which appear and disappear suddenly, and the new nice types of six and nine coexisting chaotic attractors. The computations of Lyapunov exponents confirm the dynamical behaviors. The results can be useful when the local and global stabilities in discrete-time predatorprey systems are concerned.

This paper is organized as follows. In Section 2, we show the existence and stability of fixed points. In Section 3, the sufficient conditions for the existence of codimension-one bifurcations, including flip bifurcation and Neimark-Sacker bifurcation, are obtained. In Section 4, numerical simulation results are presented to support the theoretical analysis, and they exhibit new and rich dynamical behaviors. In Section 5, chaos is controlled to an unstable fixed point using the feedback control method. A brief conclusion is given in Section 6.

\section{Existence and stability of fixed points}

For system (1), letting $\bar{u}=x$ and $\bar{v}=b y$, we obtain

$$
\left\{\begin{array}{l}
\bar{u}_{n+1}=a \bar{u}_{n}\left(1-\bar{u}_{n}\right)-\bar{u}_{n} \bar{v}_{n}, \\
\bar{v}_{n+1}=-c \bar{v}_{n}+d \bar{u}_{n} \bar{v}_{n} .
\end{array}\right.
$$

For simplicity, we will still use $x$ and $y$ instead of $\bar{u}$ and $\bar{v}$. Thus, system (2) can be rewritten as

$$
\left\{\begin{array}{l}
x_{n+1}=a x_{n}\left(1-x_{n}\right)-x_{n} y_{n} \\
y_{n+1}=-c y_{n}+d x_{n} y_{n}
\end{array}\right.
$$

We focus ourselves on the dynamical behavior of system (3).

It is easy to see that system (3) has one extinction fixed point $(0,0)$, one exclusion fixed point $\left(\frac{a-1}{a}, 0\right)$ for $a>1$, and one coexistence fixed point $\left(x^{*}, y^{*}\right)=\left(\frac{1+c}{d}, \frac{d(a-1)-a(1+c)}{d}\right)$ for $d>$ $\frac{a(1+c)}{a-1}$ and $a>1$. Thus, $\left(x^{*}, y^{*}\right)$ is the unique positive fixed point of system (3).

The following lemma confirms the stability of fixed points of system (3) under some conditions.

Lemma 2.1 For the predator-prey system (3), the following statements are true:

(i) $(0,0)$ is asymptotically stable if $0<a, c<1$; 
(ii) $\left(\frac{a-1}{a}, 0\right)$ is asymptotically stable if $1<a \leq 3$ and $\max \left\{0, \frac{a(c-1)}{a-1}\right\}<d<\frac{a(1+c)}{a-1}$;

(iii) $\left(\frac{1+c}{d}, \frac{d(a-1)-a(1+c)}{d}\right)$ is asymptotically stable if and only if one of the following conditions holds:
(a) $1<a \leq 3, c>0$ and $\frac{a(1+c)}{a-1}<d<\frac{a(2+c)}{a-1}$;
(b) $3<a \leq 5, c>0$ and $\frac{a(1+c)(3+c)}{3+a-c+a c}<d<\frac{a(2+c)}{a-1}$;
(c) $5<a<9,0<c<\frac{9-a}{a-5}$ and $\frac{a(1+c)(3+c)}{3+a-c+a c}<d<\frac{a(2+c)}{a-1}$.

Proof (i) For the fixed point $(0,0)$, the corresponding characteristic equation is $\lambda^{2}-(a-$ $c) \lambda-a c=0$, and its roots are $\lambda_{1}=a, \lambda_{2}=-c$. Hence, $(0,0)$ is asymptotically stable when $0<a, c<1$ and is unstable when $a>1$ or $c>1$.

(ii) For the exclusion fixed point $\left(\frac{a-1}{a}, 0\right)$ when $a>1$, linearizing system (3) about $\left(\frac{a-1}{a}, 0\right)$, we have the coefficient matrix

$$
J_{0}=\left(\begin{array}{cc}
2-a & -\frac{a-1}{a} \\
0 & -c+\frac{d(a-1)}{a}
\end{array}\right)
$$

Clearly, $J_{0}$ has characteristic roots $\lambda_{1}=2-a, \lambda_{2}=-c+\frac{d(a-1)}{a}$. Then $\left|\lambda_{i}\right|<1(i=1,2)$ if and only if $1<a<3$ and $\max \left\{0, \frac{a(c-1)}{a-1}\right\}<d<\frac{a(1+c)}{a-1}$.

We further will prove that when $a=3$, the exclusion fixed point $\left(\frac{a-1}{a}, 0\right)$ is asymptotically stable and when $d=\frac{a(1+c)}{a-1}$, it is unstable by using center manifold theory.

Letting $u=x-\frac{a-1}{a}$ and $v=y$ in (3), we have

$$
\left(\begin{array}{l}
u \\
v
\end{array}\right) \mapsto\left(\begin{array}{c}
(2-a) u+\frac{1-a}{a} v-a u^{2}-u v \\
\frac{d(a-1)-a c}{a} v+d u v
\end{array}\right)
$$

Now we consider the first case, that is, $a=3$ and $\max \left\{0, \frac{3(c-1)}{2}\right\}<d<\frac{3(1+c)}{2}$. System (4) becomes

$$
\left(\begin{array}{l}
u \\
v
\end{array}\right) \mapsto\left(\begin{array}{cc}
-1 & -\frac{2}{3} \\
0 & \frac{2 d-3 c}{3}
\end{array}\right)\left(\begin{array}{l}
u \\
v
\end{array}\right)+\left(\begin{array}{c}
-3 u^{2}-u v \\
d u v
\end{array}\right)
$$

Letting

$$
T=\left(\begin{array}{cc}
1 & -\frac{2}{3-3 c+2 d} \\
0 & 1
\end{array}\right)
$$

and using the translation $\left(\begin{array}{l}u \\ v\end{array}\right)=T\left(\begin{array}{l}X \\ Y\end{array}\right)$, we can rewrite the map (5) as

$$
\left(\begin{array}{c}
X \\
Y
\end{array}\right) \mapsto\left(\begin{array}{cc}
-1 & 0 \\
0 & \frac{2 d-3 c}{3}
\end{array}\right)\left(\begin{array}{l}
X \\
Y
\end{array}\right)+\left(\begin{array}{c}
\tilde{f}(X, Y) \\
\tilde{g}(X, Y)
\end{array}\right)
$$

where

$$
\begin{aligned}
& \tilde{f}(X, Y)=-3 X^{2}+\frac{3(3+c)}{3-3 c+2 d} X Y-\frac{6(1+c)}{(3-3 c+2 d)^{2}} Y^{2}, \\
& \tilde{g}(X, Y)=d X Y-\frac{2 d}{3-3 c+2 d} Y^{2} .
\end{aligned}
$$


We assume that a center manifold has the form $Y=h(X)=\tilde{\alpha} X^{2}+\tilde{\beta} X^{3}+O\left(|X|^{4}\right)$. Then it must satisfy

$$
h(-X+\tilde{f}(X, h(X)))-\frac{2 d-3 c}{3} h(X)-\tilde{g}(X, h(X))=0 .
$$

By approximate computation, for the center manifold, we obtain $\tilde{\alpha}=0$ and $\tilde{\beta}=0$. Hence, $h(X)=0$, and on the center manifold $Y=0$, the new map $\hat{f}$ is given by

$$
\hat{f}=-X+\tilde{f}(X, h(X))=-X-3 X^{2} .
$$

Some computations show that the Schwarzian derivative of this map at $X=0$ is $S(\hat{f}(0))=$ $-54<0$. Hence, by [25] the exclusion fixed point $\left(\frac{a-1}{a}, 0\right)$ is asymptotically stable.

Next we consider the second case, that is, $1<a<3$ and $d=\frac{a(1+c)}{a-1}$. System (4) becomes

$$
\left(\begin{array}{l}
u \\
v
\end{array}\right) \mapsto\left(\begin{array}{cc}
2-a & \frac{1-a}{a} \\
0 & 1
\end{array}\right)\left(\begin{array}{l}
u \\
v
\end{array}\right)+\left(\begin{array}{c}
-a u^{2}-u v \\
\frac{a(1+c)}{a-1} u v
\end{array}\right)
$$

We construct the invertible matrix

$$
T=\left(\begin{array}{cc}
1 & -\frac{1}{a} \\
0 & 1
\end{array}\right)
$$

and use the translation $\left(\begin{array}{l}u \\ v\end{array}\right)=T\left(\begin{array}{l}X \\ Y\end{array}\right)$. Then the map (7) becomes

$$
\left(\begin{array}{l}
X \\
Y
\end{array}\right) \mapsto\left(\begin{array}{cc}
2-a & 0 \\
0 & 1
\end{array}\right)\left(\begin{array}{l}
X \\
Y
\end{array}\right)+\left(\begin{array}{l}
\tilde{f}_{1}(X, Y) \\
\tilde{g}_{1}(X, Y)
\end{array}\right)
$$

where $\tilde{f}_{1}(X, Y)=-a X^{2}+\frac{a+c}{a-1} X Y-\frac{1+c}{a(a-1)} Y^{2}$ and $\tilde{g}_{1}(X, Y)=\frac{a(1+c)}{a-1} X Y-\frac{1+c}{a-1} Y^{2}$.

Consider a center manifold with the form $X=h(Y)=\tilde{\alpha}_{1} Y^{2}+\tilde{\beta}_{1} Y^{3}+O\left(|Y|^{4}\right)$. Then it must satisfy

$$
h\left(Y+\tilde{g}_{1}(h(Y), Y)\right)-(2-a) h(Y)-\tilde{f}_{1}(h(Y), Y)=0 .
$$

By approximate computation, for the center manifold, we obtain $\tilde{\alpha}_{1}=-\frac{1+c}{a(a-1)^{2}}$ and $\tilde{\beta}_{1}=-\frac{(1+c)(2+a+3 c)}{a(a-1)^{4}}$. Hence, $h(Y)=-\frac{1+c}{a(a-1)^{2}} Y^{2}-\frac{(1+c)(2+a+3 c)}{a(a-1)^{4}} Y^{3}+O\left(|Y|^{4}\right)$, and on the center manifold $X=h(Y)$, the new map $\hat{f}_{1}$ is given by

$$
\hat{f}_{1}=Y+\tilde{g}_{1}(h(Y), Y)=Y-\frac{1+c}{a-1} Y^{2}-\frac{(1+c)^{2}}{(a-1)^{3}} Y^{3}+O\left(|Y|^{4}\right) \text {. }
$$

Computations show that $\hat{f}_{1}^{\prime}(0)=1$ and $\hat{f}_{1}^{\prime \prime}(0)=-\frac{2(1+c)}{a-1}<0$. Hence, by [25] the exclusion fixed point $\left(\frac{a-1}{a}, 0\right)$ is unstable. More precisely, it is a semistable fixed point from the right.

Therefore, $\left(\frac{a-1}{a}, 0\right)$ is asymptotically stable when $1<a \leq 3$ and $\max \left\{0, \frac{a(c-1)}{a-1}\right\}<d<\frac{a(1+c)}{a-1}$.

(iii) Finally, we consider the positive fixed point $\left(x^{*}, y^{*}\right)=\left(\frac{1+c}{d}, \frac{d(a-1)-a(1+c)}{d}\right)$ for $d>\frac{a(1+c)}{a-1}$ $(a>1)$. The Jacobian matrix evaluated at the positive fixed point $\left(x^{*}, y^{*}\right)$ is given by

$$
J^{*}=\left(\begin{array}{cc}
\frac{d-a(1+c)}{d} & -\frac{1+c}{d} \\
d(a-1)-a(1+c) & 1
\end{array}\right)
$$


and the characteristic equation of the Jacobian matrix $J^{*}$ can be written as

$$
P^{*}(\lambda)=\lambda^{2}-\left(\operatorname{tr} J^{*}\right) \lambda+\operatorname{det} J^{*}=\lambda^{2}+\frac{a+a c-2 d}{d} \lambda-\frac{a(1+c)(2+c-d)+c d}{d}=0 .
$$

According to the Jury conditions [9], in order to find the asymptotically stable region of $\left(x^{*}, y^{*}\right)$, we need to find the region that satisfies the following conditions:

$$
P^{*}(1)>0, \quad P^{*}(-1)>0 \quad \text { and } \quad \operatorname{det} J^{*}<1 .
$$

Since

$$
\begin{aligned}
& P^{*}(1)=-\frac{(1+c)(a+a c+d-a d)}{d}, \\
& P^{*}(-1)=-\frac{3 a+4 a c+a c^{2}-3 d-a d+c d-a c d}{d}, \text { and } \\
& \operatorname{det} J^{*}=-\frac{a(1+c)(2+c-d)+c d}{d},
\end{aligned}
$$

from the relations $P^{*}(1)>0, P^{*}(-1)>0$, and $\operatorname{det} J^{*}<1$ we have that

$$
\begin{aligned}
& 1<a \leq 3, \quad c>0, \quad \frac{a(1+c)}{a-1}<d<\frac{a(2+c)}{a-1}, \quad \text { or } \\
& 3<a \leq 5, \quad c>0, \quad \frac{3 a+4 a c+a c^{2}}{3+a-c+a c}<d<\frac{a(2+c)}{a-1}, \quad \text { or } \\
& 5<a<9, \quad 0<c<\frac{9-a}{a-5}, \quad \frac{3 a+4 a c+a c^{2}}{3+a-c+a c}<d<\frac{a(2+c)}{a-1} .
\end{aligned}
$$

This completes the proof of Lemma 2.1.

\section{Bifurcations}

In this section, we mainly focus on the flip bifurcation and Neimark-Sacker bifurcation of the positive fixed point $\left(x^{*}, y^{*}\right)$. We choose the parameter $d$ as a bifurcation parameter for analyzing the flip bifurcation and Neimark-Sacker bifurcation of $\left(x^{*}, y^{*}\right)$ by using the center manifold theorem and bifurcation theory of [23, 24].

First, we have the following result on the flip bifurcation of system (3).

Theorem 3.1 System (3) undergoes a flip bifurcation at $\left(x^{*}, y^{*}\right)$ if the following conditions are satisfied: $c>0, a>3, a \neq \frac{9+5 c}{1+c}$, and $d=\frac{a(1+c)(3+c)}{3+a-c+a c}$. Moreover, if $3<a<\frac{9+5 c}{1+c}$, then period -2 points that bifurcate from this fixed point are unstable.

Proof If $d^{*}=\frac{a(1+c)(3+c)}{3+a-c+a c}$, then the eigenvalues of the fixed point $\left(x^{*}, y^{*}\right)$ are $\lambda_{1}=-1$ and $\lambda_{2}=$ $\frac{6-a+4 c-a c}{3+c}$. The condition $\left|\lambda_{2}\right| \neq 1$ leads to $a \neq 3, \frac{9+5 c}{1+c}$. In addition, note that the existence of the positive fixed point is assured by the relation $d>\frac{a(1+c)}{a-1}(a>1)$, so we get $a>3$. Hence, we further assume that $a>3$ and $a \neq \frac{9+5 c}{1+c}$.

Let $u=x-x^{*}, v=y-y^{*}$, and $\bar{d}=d-d^{*}$. We consider the parameter $\bar{d}$ as a new and dependent variable. Then the map (3) becomes

$$
\left(\begin{array}{l}
u \\
\bar{d} \\
v
\end{array}\right) \mapsto\left(\begin{array}{ccc}
\frac{2 c-a(1+c)}{3+c} & 0 & \frac{c-a(1+c)-3}{a(3+c)} \\
0 & 1 & 0 \\
\frac{2 a(a-3)(1+c)}{3+a-c+a c} & \frac{2(a-3)(3-c+a+a c)}{a(3+c)^{2}} & 1
\end{array}\right)\left(\begin{array}{l}
u \\
\bar{d} \\
v
\end{array}\right)+\left(\begin{array}{c}
f_{1}(u, \bar{d}, v) \\
0 \\
f_{2}(u, \bar{d}, v)
\end{array}\right),
$$


where

$$
\begin{aligned}
& f_{1}(u, \bar{d}, v)=-a u^{2}-u v \\
& f_{2}(u, \bar{d}, v)=\frac{a(1+c)(3+c)}{3+a-c+a c} u v+\frac{2(a-3)}{3+c} u \bar{d}+\frac{3+a-c+a c}{a(3+c)} v \bar{d}+u v \bar{d} .
\end{aligned}
$$

Let

$$
T=\left(\begin{array}{ccc}
-\frac{3+a-c+a c}{a(a-3)(1+c)} & -\frac{1}{a} & -\frac{3+a-c+a c}{2 a(3+c)} \\
0 & \frac{a(1+c)(3+c)^{2}}{(3+a-c+a c)^{2}} & 0 \\
1 & 1 & 1
\end{array}\right),
$$

and use the translation $\left(\begin{array}{l}u \\ d \\ v\end{array}\right)=T\left(\begin{array}{l}X \\ \mu \\ Y\end{array}\right)$. Then the map (10) becomes

$$
\left(\begin{array}{c}
X \\
\mu \\
Y
\end{array}\right) \mapsto\left(\begin{array}{ccc}
-1 & 0 & 0 \\
0 & 1 & 0 \\
0 & 0 & \frac{6+4 c-a(1+c)}{3+c}
\end{array}\right)\left(\begin{array}{l}
X \\
\mu \\
Y
\end{array}\right)+\left(\begin{array}{c}
F_{1}(X, \mu, Y) \\
0 \\
F_{2}(X, \mu, Y)
\end{array}\right)
$$

where

$$
\begin{aligned}
& F_{1}(X, \mu, Y)=-\frac{(3+c)\left(9-2 c-3 c^{2}+a(1+c)^{2}\right) X^{2}}{(a-3)(1+c)(a-9-5 c+a c)}-\frac{(3+c)(3+a-c+a c) X Y}{2(a-9-5 c+a c)} \\
& -\frac{(a-3)(a+c)(1+c)^{2} Y^{2}}{2(3+c)(a-9-5 c+a c)}-\frac{2(a-3)^{2}(1+c)^{2}(3+c) \mu^{2}}{(a-9-5 c+a c)(3+a-c+a c)^{2}} \\
& -\frac{(3+c)^{2} X \mu}{a-9-5 c+a c}-\frac{(a-3)(1+c)^{2}(9+c-a(5+c)) Y \mu}{2\left(27+6 c-5 c^{2}+6 a(1+c)^{2}-a^{2}(1+c)^{2}\right)} \\
& -\frac{(a-3)(1+c)^{2}(3+c) Y^{2} \mu}{2(a-9-5 c+a c)(3+a-c+a c)}-\frac{(1+c)(3+c) X Y \mu}{2(a-9-5 c+a c)} \\
& -\frac{(1+c)(3+c)^{2} X^{2} \mu}{(a-9-5 c+a c)(3+a-c+a c)}-\frac{2(1+c)(3+c)^{2}(a-2 c+a c) X \mu^{2}}{(a-9-5 c+a c)(3+a-c+a c)^{2}} \\
& -\frac{(a-3)(1+c)^{2}(3+c)(9+a+c+a c) Y \mu^{2}}{2(a-9-5 c+a c)(3+a-c+a c)^{2}} \\
& -\frac{(a-3)(1+c)^{2}(3+c)^{2} \mu^{3}}{(a-9-5 c+a c)(3+a-c+a c)^{2}} \\
& F_{2}(X, \mu, Y)=\frac{2(3+c)^{3} X^{2}}{(a-3)(1+c)(a-9-5 c+a c)}+\frac{\left(c(3-c)+a^{2}(1+c)+a\left(3+c^{2}\right)\right) X Y}{(a-3)(a-9-5 c+a c)} \\
& +\frac{(1+c)\left(27+21 c+2 c^{2}-6 a(1+c)+a^{2}(1+c)\right) Y^{2}}{2(3+c)(a-9-5 c+a c)} \\
& +\frac{2(3+c)^{2}(a(5+c)-9-c) X \mu}{(a-3)\left(-27-6 c+5 c^{2}-6 a(1+c)^{2}+a^{2}(1+c)^{2}\right)} \\
& +\frac{4(a-3)(1+c)(3+c)^{2} \mu^{2}}{(a-9-5 c+a c)(3+a-c+a c)^{2}}+\frac{(1+c)(a+c) Y \mu}{a-9-5 c+a c} \\
& +\frac{(1+c)(3+c)^{2} Y^{2} \mu}{(a-9-5 c+a c)(3+a-c+a c)}+\frac{(3+c)^{2} X Y \mu}{(a-3)(a-9-5 c+a c)}
\end{aligned}
$$




$$
\begin{aligned}
& +\frac{2(3+c)^{3} X^{2} \mu}{(a-3)(a-9-5 c+a c)(3+a-c+a c)} \\
& +\frac{4(3+c)^{3}(a-2 c+a c) X \mu^{2}}{(a-3)(a-9-5 c+a c)(3+a-c+a c)^{2}} \\
& +\frac{(1+c)(3+c)^{2}(9+a+c+a c) Y \mu^{2}}{(a-9-5 c+a c)(3+a-c+a c)^{2}} \\
& +\frac{2(1+c)(3+c)^{3} \mu^{3}}{(a-9-5 c+a c)(3+a-c+a c)^{2}} .
\end{aligned}
$$

By the center manifold theorem we know that the stability of $(X, Y)=(0,0)$ near $\mu=0$ can be determined by studying a one-parameter family of maps on a center manifold, which can be represented as follows:

$$
W^{c}(0)=\left\{(X, \mu, Y) \in R^{3} \mid Y=h^{*}(X, \mu), h^{*}(0,0)=0, D h^{*}(0,0)=0\right\} .
$$

Assume that

$$
h^{*}(X, \mu)=\alpha X^{2}+\beta X \mu+\gamma \mu^{2}+O\left((|X|+|\mu|)^{3}\right) .
$$

By approximate computation for the center manifold, we obtain

$$
\begin{aligned}
& \alpha=\frac{2(3+c)^{4}}{(a-3)^{2}(1+c)^{2}(a-9-5 c+a c)}, \\
& \beta=\frac{2(3+c)^{3}(5 a-9-c+a c)}{(a-3)(3+a-c+a c)(a-9-5 c+a c)^{2}}, \\
& \gamma=\frac{4(3+c)^{3}}{(a-9-5 c+a c)(3+a-c+a c)^{2}} .
\end{aligned}
$$

Thus, the map restricted to the center manifold is given by

$$
\begin{aligned}
\tilde{F}: X \rightarrow & -X+h_{1} X^{2}+h_{2} X \mu+h_{3} \mu^{2}+h_{4} X^{3}+h_{5} X^{2} \mu+h_{6} X \mu^{2}+h_{7} \mu^{3} \\
& +O\left((|X|+|\mu|)^{4}\right),
\end{aligned}
$$

where

$$
\begin{aligned}
& h_{1}=-\frac{(3+c)\left(9-2 c-3 c^{2}+a(1+c)^{2}\right)}{(a-3)(1+c)(a-9-5 c+a c)}, \\
& h_{2}=-\frac{(3+c)^{2}}{a-9-5 c+a c} \\
& h_{3}=-\frac{2(a-3)^{2}(1+c)^{2}(3+c)}{(a-9-5 c+a c)(3+a-c+a c)^{2}}, \\
& h_{4}=-\frac{(3+c)^{5}(3+a-c+a c)}{(a-3)^{2}(1+c)^{2}(a-9-5 c+a c)^{2}}, \\
& h_{5}=-\frac{(1+c)(3+c)^{2}\left(-81-36 c-5 c^{2}-2 c^{3}+a^{2}(1+c)^{2}+2 a\left(36+25 c+6 c^{2}+c^{3}\right)\right)}{(3+a-c+a c)(a-9-5 c+a c)^{3}}, \\
& h_{6}=(3+c)^{2}\left(243+135 c+186 c^{2}+234 c^{3}+67 c^{4}-c^{5}-2 a^{3}(1+c)^{4}\right.
\end{aligned}
$$




$$
\begin{aligned}
+ & \left.a(1+c)^{2}\left(216-57 a-6 c-7 a c-44 c^{2}+9 a c^{2}+2 c^{3}-a c^{3}\right)\right) \\
& /(3+a-c+a c)^{2}(a-9-5 c+a c)^{3}, \\
h_{7}= & -\frac{(a-3)^{2}\left(3+4 c+c^{2}\right)^{2}\left(27+10 c-c^{2}+a(1+c)^{2}\right)}{(a-9-5 c+a c)^{2}(3+a-c+a c)^{3}} .
\end{aligned}
$$

If the map (11) undergoes a flip bifurcation, then it must satisfy the following conditions:

$$
\alpha_{1}=\left.\left[\frac{\partial F}{\partial \mu} \cdot \frac{\partial^{2} F}{\partial X^{2}}+2 \frac{\partial^{2} F}{\partial X \partial \mu}\right]\right|_{(0,0)} \neq 0
$$

and

$$
\alpha_{2}=\left.\left[\frac{1}{2} \cdot\left(\frac{\partial^{2} F}{\partial X^{2}}\right)^{2}+\frac{1}{3} \cdot \frac{\partial^{3} F}{\partial X^{3}}\right]\right|_{(0,0)} \neq 0
$$

By a simple calculation we obtain

$$
\alpha_{1}=-\frac{2(3+c)^{2}}{a-9-5 c+a c} \neq 0 \quad \text { for } c>0, a>3 \text { and } a \neq \frac{9+5 c}{1+c}
$$

and

$$
\alpha_{2}=\frac{2(3+c)^{2}\left(a(1+c)^{3}-2 c\left(c^{2}-5\right)\right)}{(a-3)^{2}(1+c)^{2}(a-9-5 c+a c)} \neq 0 \quad \text { for } c>0, a>3 \text { and } a \neq \frac{9+5 c}{1+c}
$$

It is easy to check that if $3<a<\frac{9+5 c}{1+c}$, then $\left|\lambda_{2}\right|<1$ and $\alpha_{2}<0$. Thus, period-2 points that bifurcate from this fixed point are unstable.

This completes the proof of Theorem 3.1.

For Neimark-Sacker bifurcation, we have the following theorem.

Theorem 3.2 System (3) undergoes a Neimark-Sacker bifurcation at the fixed point $\left(x^{*}, y^{*}\right)$ if the following conditions are satisfied: $c>0,1<a<9, a \neq \frac{5+3 c}{1+c}, \frac{7+4 c}{1+c}$, and $d=\bar{d}^{*}=\frac{a(2+c)}{a-1}$. Moreover, $k<0$, and thus an attracting invariant closed curve bifurcates from the fixed point for $d>\bar{d}^{*}$.

Proof The characteristic equation associated with the linearized system (3) at the fixed point $\left(x^{*}(d), y^{*}(d)\right)$ is given by

$$
\lambda^{2}+p(d) \lambda+q(d)=0
$$

The eigenvalues of the characteristic equation (12) are given as

$$
\lambda_{1,2}(d)=\frac{-p(d) \pm \sqrt{p(d)^{2}-4 q(d)}}{2}
$$

where $p(d)=c-a+(2 a-d) x^{*}+y^{*}$ and $q(d)=-a c+a(2 c+d) x^{*}-2 a d x^{* 2}+c y^{*}$. 
The eigenvalues $\lambda_{1,2}$ are complex conjugates for $p(d)^{2}-4 q(d)<0$, which leads to

$$
d>\frac{a(1+c)+\sqrt{a^{2}\left(c+c^{2}\right)+a^{3}(1+c)}}{2(a-1)} .
$$

Let

$$
\bar{d}^{*}=\frac{a(2+c)}{a-1} \quad \text { for } 1<a<9 .
$$

We get $q(d)=1$ and $\lambda_{1,2}=\frac{5-a+3 c-a c}{2(2+c)} \pm \frac{i \sqrt{(1-a)(1+c)(a-9-5 c+a c)}}{2(2+c)}=\rho \pm i \omega$. Under condition (14), we have

$$
\left|\lambda_{1,2}(d)\right|=(q(d))^{\frac{1}{2}} \quad \text { and } \quad d_{1}=\left.\frac{d\left|\lambda_{1,2}(d)\right|}{d d}\right|_{d=\bar{d}^{*}}=\frac{(a-1)^{2}(1+c)}{2 a(2+c)} \neq 0 .
$$

In addition, if $p\left(\bar{d}^{*}\right) \neq 0,1$, which leads to

$$
a \neq \frac{5+3 c}{1+c} \text { and } a \neq \frac{7+4 c}{1+c}
$$

then we obtain that $\lambda_{1,2}^{n}\left(\bar{d}^{*}\right) \neq 1(n=1,2,3,4)$.

Letting $u=x-x^{*}$ and $v=y-y^{*}$, the map (3) becomes

$$
\left(\begin{array}{l}
u \\
v
\end{array}\right) \mapsto\left(\begin{array}{cc}
\frac{3+2 c-a(1+c)}{2+c} & -\frac{(a-1)(1+c)}{a(2+c)} \\
a & 1
\end{array}\right)\left(\begin{array}{l}
u \\
v
\end{array}\right)+\left(\begin{array}{l}
f_{1}(u, v) \\
f_{2}(u, u)
\end{array}\right)
$$

where $f_{1}(u, v)=-a u^{2}-u v$ and $f_{2}(u, v)=\frac{a(2+c)}{a-1} u v$.

Let

$$
T=\left(\begin{array}{cc}
-\frac{\sqrt{(1-a)(1+c)(a-9-5 c+a c)}}{2 a(2+c)} & \frac{1+c-a-a c}{2 a(2+c)} \\
0 & 1
\end{array}\right)
$$

and use the translation $\left(\begin{array}{l}u \\ v\end{array}\right)=T\left(\begin{array}{l}X \\ Y\end{array}\right)$. Then the map (15) becomes

$$
\left(\begin{array}{c}
X \\
Y
\end{array}\right) \mapsto\left(\begin{array}{cc}
\rho & -\omega \\
\omega & \rho
\end{array}\right)\left(\begin{array}{l}
X \\
Y
\end{array}\right)+\left(\begin{array}{l}
F_{1}(X, Y) \\
F_{2}(X, Y)
\end{array}\right)
$$

where

$$
\begin{aligned}
F_{1}(X, Y)= & \frac{\sqrt{(1-a)(1+c)(a-9-5 c+a c)}}{2(2+c)} X^{2}-\frac{4+c-c^{2}-2 a(1+c)}{2(2+c)} X Y \\
& -\frac{(a-1)(1+c)\left(a-3+a c+c^{2}\right)}{2(2+c) \sqrt{(1-a)(1+c)(a-9-5 c+a c)}} Y^{2}, \\
F_{2}(X, Y)= & -\frac{\sqrt{(1-a)(1+c)(a-9-5 c+a c)}}{2(a-1)} X Y-\frac{1+c}{2} Y^{2} .
\end{aligned}
$$

Notice that (16) is exactly in the form on the center manifold in which the coefficient $k$ [23] is given by

$$
k=-\operatorname{Re}\left[\frac{(1-2 \lambda) \bar{\lambda}^{2}}{1-\lambda} \xi_{11} \xi_{20}\right]-\frac{1}{2}\left|\xi_{11}\right|^{2}-\left|\xi_{02}\right|^{2}+\operatorname{Re}\left(\bar{\lambda} \xi_{21}\right)
$$


where

$$
\begin{aligned}
& \xi_{20}=\frac{1}{8}\left[\left(F_{1 X X}-F_{1 Y Y}+2 F_{2 X Y}\right)+i\left(F_{2 X X}-F_{2 Y Y}-2 F_{1 X Y}\right)\right], \\
& \xi_{11}=\frac{1}{4}\left[\left(F_{1 X X}+F_{1 Y Y}\right)+i\left(F_{2 X X}+F_{2 Y Y}\right)\right], \\
& \xi_{02}=\frac{1}{8}\left[\left(F_{1 X X}-F_{1 Y Y}-2 F_{2 X Y}\right)+i\left(F_{2 X X}-F_{2 Y Y}+2 F_{1 X Y}\right)\right], \\
& \xi_{21}=\frac{1}{16}\left[\left(F_{1 X X X}+F_{1 X Y Y}+F_{2 X X Y}+F_{2 Y Y Y}\right)+i\left(F_{2 X X X}+F_{2 X Y Y}-F_{1 X X Y}-F_{1 Y Y Y}\right)\right] .
\end{aligned}
$$

Thus, a complex calculation gives

$$
k=-\frac{A}{8(a-1)(2+c)^{3}}<0 \quad \text { for } 1<a<9,
$$

where $A=3+14 c+14 c^{2}+4 c^{3}+a^{4}(1+c)^{3}+a^{3}(1+c)^{2}\left(c^{2}-3 c-6\right)-3 a^{2}(1+c)^{2}\left(c^{2}-c-4\right)+$ $a\left(6+19 c+35 c^{2}+31 c^{3}+11 c^{4}+c^{5}\right)$.

Thus the fixed point $(X, Y)=(0,0)$ is a Neimark-Sacker bifurcation point for the map (16). This completes the proof.

\section{Numerical simulations}

In this section, numerical simulations are given, including bifurcation diagrams, Lyapunov exponents, and fractal dimension and phase portraits, to illustrate the above theoretical analysis and to show new and more complex dynamic behaviors in system (3).

The fractal dimension [26-29] is defined by using Lyapunov exponents as follows:

$$
d_{L}= \begin{cases}0 & \text { if no such } j \text { exists } \\ j+\frac{\sum_{i=1}^{i=j} L_{i}}{L_{j}} & \text { if } j<n \\ n & \text { if } j=n\end{cases}
$$

with $L_{1}, L_{2}, \ldots, L_{n}$ being Lyapunov exponents, where $j$ is the largest integer such that $\sum_{i=1}^{i=j} L_{i} \geq 0$ and $\sum_{i=1}^{i=j+1} L_{i}<0$.

Our model is a two-dimensional map that has the fractal dimension of the form

$$
d_{L}=1+\frac{L_{1}}{\left|L_{2}\right|}, \quad \text { where } L_{1}>0>L_{2} \text { and } L_{1}+L_{2}<0 .
$$

\subsection{Numerical simulations for stability and bifurcations of fixed points}

We consider the following two cases.

Case 1. A bifurcation diagram of system (3) in $(d, x)$ plane for $1.4 \leq d \leq 2.4$ and $a=3.5$ with initial value $(0.6,0.3)$ is given in Figure 1(a). It shows that there is a flip bifurcation (labeled 'P-D') emerging from the fixed point $(0.625,0.3125)$ with $d=1.92, \alpha_{1}=3.53103$, and $\alpha_{2}=-78.7813<0$.

Case 2. A bifurcation diagram of system (3) in $(d, x)$ plane is displayed in Figure 1(b) for $3 \leq d \leq 5$ and $a=2.5$ with initial value $(0.3,0.6)$. Figure $1(\mathrm{~b})$ exhibits a Neimark-Sacker bifurcation (labeled ' NS'), which occurs at fixed point $(0.32727,0.68182)$ and $d=3.66667$ with $d_{1}=0.24546>0$ and $k=-0.52652<0$. Figures 1 (a) and 1 (b) show the correctness of Theorems 3.1 and 3.2. 


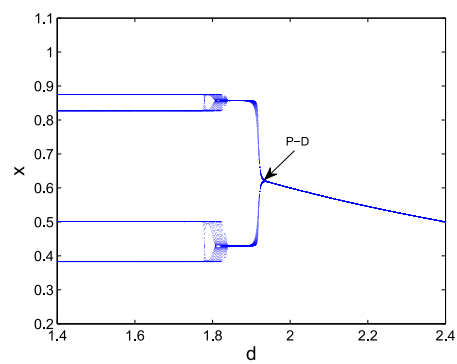

(a)

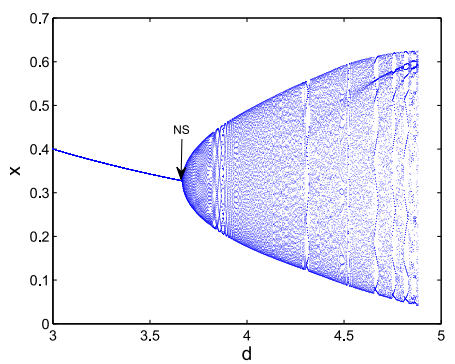

(b)

Figure 1 Bifurcation diagrams for system (3). (a) Bifurcation diagram of system $(3)$ in $(d, x)$ plane for $a=3.5$, $c=0.2, d \in(1.4,2.4)$, and the initial value $(0.6,0.3)$. (b) Bifurcation diagram of system $(3)$ in $(d, x)$ plane for $a=2.5, c=0.2, d \in(3,5)$, and the initial value $(0.3,0.6)$

\subsection{Further numerical simulations for system (3)}

In this subsection, new and interesting dynamical behaviors are investigated as the parameters vary.

The bifurcation diagrams in the two-dimensional plane are considered in the following four cases:

(i) Varying $d$ in the range $0 \leq d \leq 4.5$ and fixing $a=3.4, c=0.2$;

(ii) Varying $d$ in the range $0 \leq d \leq 4.5$ and fixing $a=3.6, c=0.2$;

(iii) Varying $d$ in the range $2.7 \leq d \leq 3.5$ and fixing $a=4.1, c=0.2$;

(iv) Varying $a$ in the range $0 \leq a \leq 4.3$ and fixing $d=3.5, c=0.2$.

Case (i). The bifurcation diagrams of system (3) in $(d, x)$ plane and in $(d, y)$ plane for $a=3.4$ and $c=0.2$ with initial value $(0.4,1.0)$ are given in Figures 2(a) and 2(c), respectively, which show the dynamical changes of the prey and predator as $d$ varies. From Figures 2(a) and 2(c) we can see that a Neimark-Sacker bifurcation emerges at $d \sim 3.1$ and an attracting invariant cycle bifurcates from the fixed point since $k=-0.69494$ and $d_{1}=0.462032$ by Theorem 3.2. We also observe the period- $6,-11,-24,-27$ windows within the chaotic regions and boundary crisis at $d=4.19$. The phase portraits corresponding to Figure 2(a) are shown in Figures 2(d)-(f) for showing eleven- and six-coexisting chaotic attractors at $d=3.58$ and 3.72 and a chaotic attractor at $d=3.8$. The maximum Lyapunov exponents corresponding to Figure 2(a) are computed in Figure 2(b). When $d=3.72$, the maximum Lyapunov exponent is $0.015>0$, which confirms the existence of the chaotic sets.

Case (ii). The bifurcation diagram of system (3) in $(d, x)$ plane for $a=3.6$ and $c=0.2$ with initial value $(0.4,1.1)$ is disposed in Figure 3(a). The maximum Lyapunov exponents corresponding to Figure 3(a) are calculated in Figure 3(b), confirming the existences of chaotic regions and period orbits as the parameter $d$ varying. Figures 3(a) and 3(b) clearly depict two onsets of chaos at $d=0$ and $d \sim 3.195$, respectively, which are the crisis. The nonattracting chaotic set at $d=1.9$ and chaotic attractor at $d=3.7$ are shown in Figures 3(e) and 3(f), respectively. Figure 3(c) is the bifurcation diagram in $(d, y)$ for $a=3.6$ and $c=0.2$, which shows the dynamical changes of the predator as $d$ varies. Comparing Figures 3(a) and 3(c), we see that there are similar dynamics for $d \in(2,4.1)$, but when the prey is in chaotic dynamic for $d \in(0,2)$, the predator tends to extinct (we also can see phase portrait Figure 3(d)).

Case (iii). The bifurcation diagram of system (3) in $(d, x)$ plane for $a=4.1$ and $c=0.2$ with initial value $(0.4,1.4)$ is shown in Figure 4(a). Figures 4(d) and 4(e) show the local amplifi- 


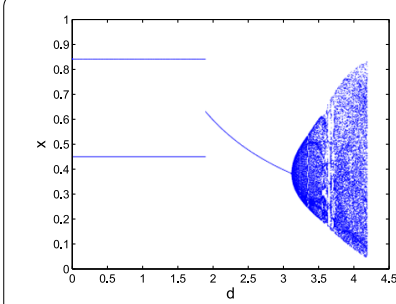

(a)

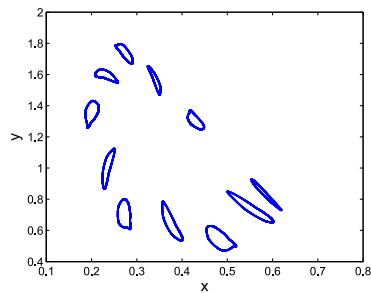

(d)

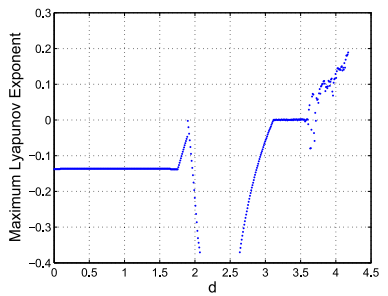

(b)

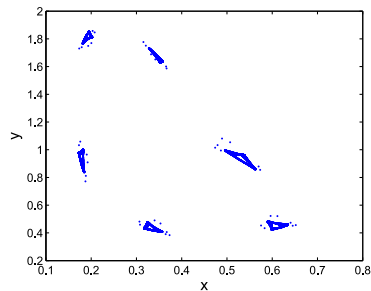

(e)

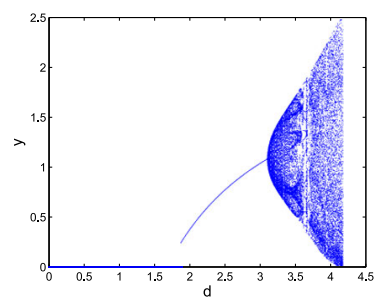

(c)

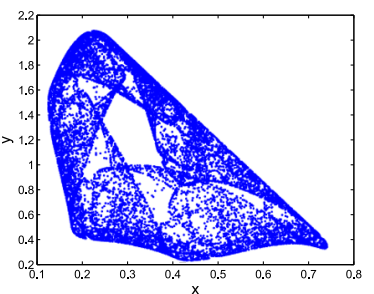

(f)

Figure 2 Bifurcation diagrams, maximum Lyapunov exponent, and phase portraits for system (3). (a) Bifurcation diagram of system (3) in $(d, x)$ plane for $a=3.4$ and $c=0.2$. (b) Maximum Lyapunov exponents corresponding to (a). (c) Bifurcation diagram in $(d, y)$ plane for $a=3.4$ and $c=0.2$. (d)-(f) Phase portraits for $d=3.58,3.72,3.8$.

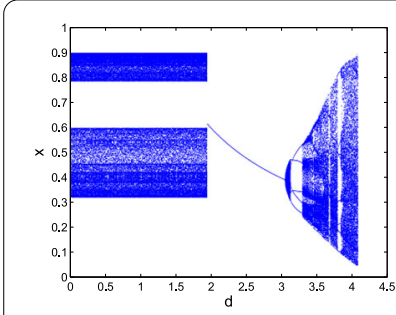

(a)

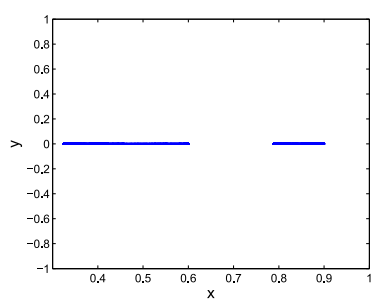

(d)

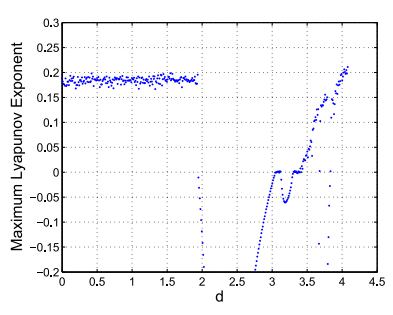

(b)

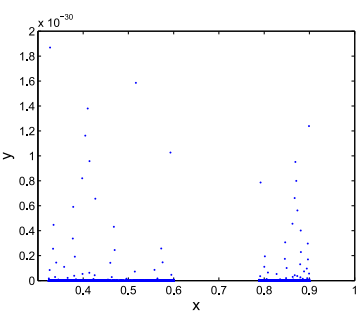

(e)

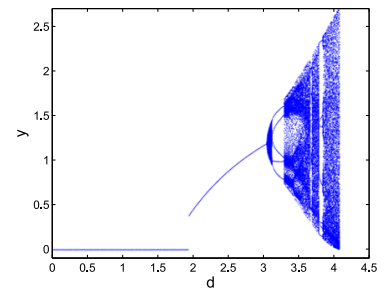

(c)

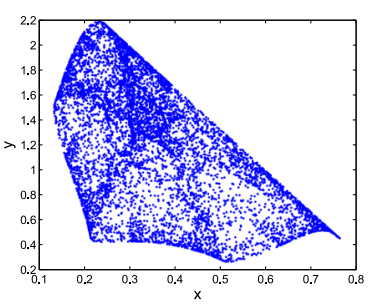

(f)

Figure 3 Bifurcation diagrams, maximum Lyapunov exponent, and phase portraits for system (3).

(a) Bifurcation diagram in $(d, x)$ plane for $a=3.6$ and $c=0.2$. (b) Maximum Lyapunov exponents corresponding to (a). (c) Bifurcation diagram in $(d, y)$ plane for $a=3.6$ and $c=0.2$. (d)-(f) Phase portraits for various values of $d$ : (d) $d=1.0$, (e) $d=1.9$, and (f) $d=3.7$.

cations for $d \in(3.08,3.24)$ and $d \in(3.2,3.23)$ in (a), respectively. The diagrams show that there is a stable fixed point for $d \in(2.7,2.9097)$ and the fixed point loses its stability as $d$ increases. Neimark-Sacker bifurcation occurs at $d \sim 2.9097$, and invariant circle appears as $d$ increases, and the invariant circle suddenly becomes to period-9 orbits at $d \sim 3.085$. 


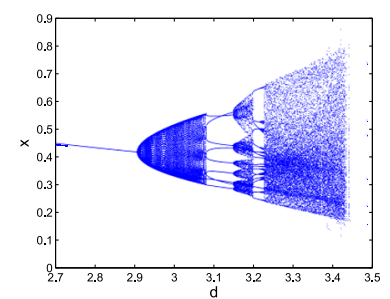

(a)

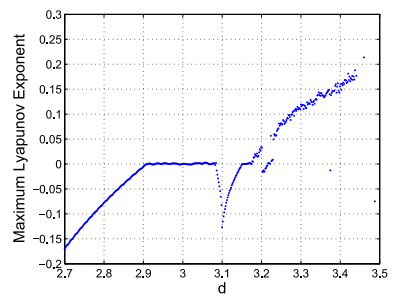

(b)

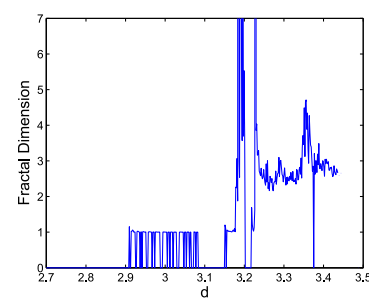

(c)

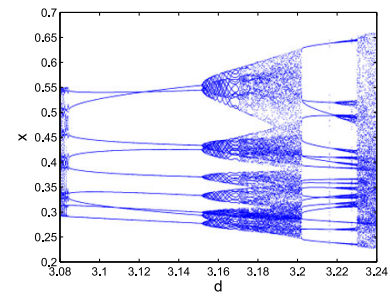

(d)

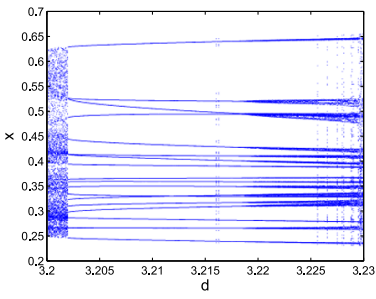

(e)

Figure 4 Bifurcation diagrams, maximum Lyapunov exponent, and fractal dimensions for system (3). (a) Bifurcation diagram in $(d, x)$ plane for $a=4.1$ and $c=0.2$. (b) Maximum Lyapunov exponents corresponding to (a). (c) Fractal dimensions corresponding to (a). Local amplification corresponding to (a) for (d) $d \in(3.08,3.24)$ and (e) $d \in(3.2,3.25)$.

Then, as $d$ grows, chaos appears at $d \sim 3.16$, the chaotic behavior disappears suddenly and transforms to period-18 orbits at $d \sim 3.203$. Particularly, the chaotic behavior disappears suddenly and becomes to period-11 orbits at $d \sim 3.3741$. The phase portraits for various values of $d$ are shown in Figures 5(a)-(i). From Figure 5 we observe that there are period-9 and -11 orbits, nine-coexisting chaotic attractors, and attracting chaotic sets.

The maximum Lyapunov exponents and fractal dimension corresponding to (a) are given in Figures 4(b) and 4(c), respectively. The maximum Lyapunov exponents are negative for the parameter $d \in(2.7,2.91)$, whereas the fixed point is stable. For $d \in(2.91,3.17)$, the maximum Lyapunov exponents are in the neighborhood of zero, which corresponds to quasi-period solutions or coexistence of chaos and quasi-period solutions. For $d \in$ $(3.17,3.5)$, the maximum Lyapunov exponents are positive with a few negative, which shows that a period window occurs in the chaotic region.

Case (iv). The bifurcation diagram of system (3) in $(a, x)$ plane for $d=3.5$ and $c=0.2$ with initial value $(0.3,0.7)$ is shown in Figure 6(a). Figures 6(d) and 6(e) are the local amplifications for $a \in(3.4,3.72)$ and $a \in(3.5,3.7)$ in (a). The maximum Lyapunov exponents and fractal dimension corresponding to (a) are plotted in Figures 6(b) and 6(c), respectively. From Figure 6(b) we see that some Lyapunov exponents are greater than 0 , some are smaller than 0 ,and thus there exist stable fixed points or period windows in the chaotic region. The diagrams show that there is a stable fixed point for $a \in(1.8,2.69)$, and the fixed point loses its stability as $a$ increases. Neimark-Sacker bifurcation occurs at $a \sim 2.69$, and invariant circle appears as $a$ increases, and the invariant circle suddenly becomes to period- 6 orbits at $a \sim 2.93$ and period-11 orbits at $a \sim 3.35$. Furthermore, as $a$ grows, we can observe the period-6, $-16,-20,-21,-27$, and -37 windows within the chaotic regions and boundary crisis at $a=4.09$. The phase portraits for various values of $a$ are shown in 


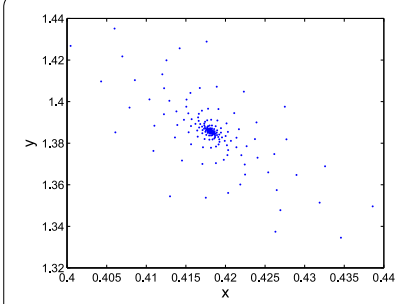

(a) $d=2.87$

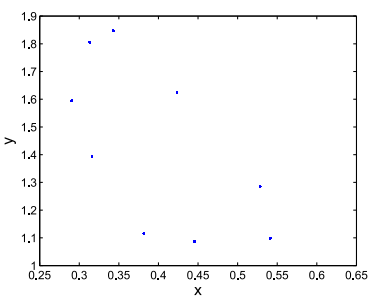

(d) $d=3.1$

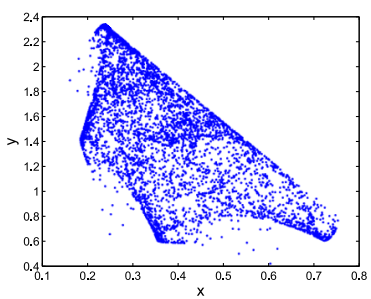

(g) $d=3.37$

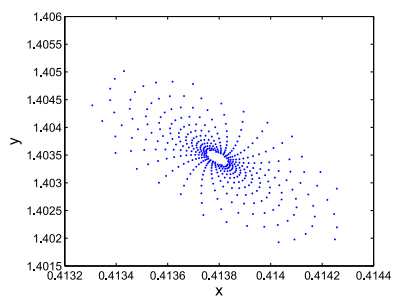

(b) $d=2.9$

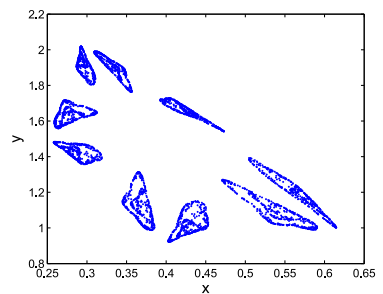

(e) $d=3.19$

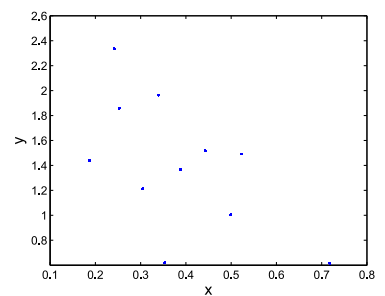

(h) $d=3.3743$

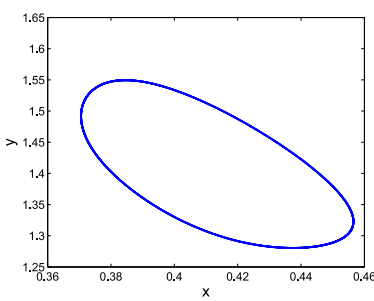

(c) $d=2.93$

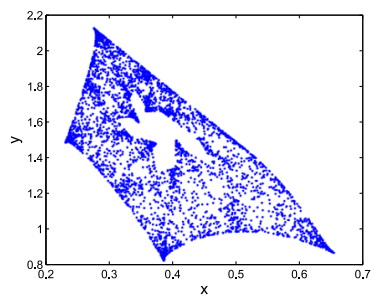

(f) $d=3.23$

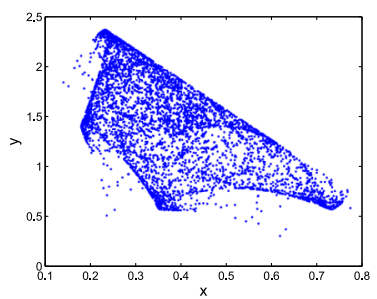

(i) $d=3.39$

Figure 5 Phase portraits for various values of $d$ corresponding to Figure 4(a).

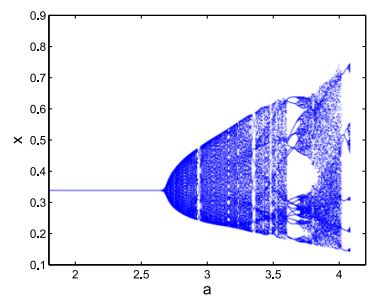

(a)

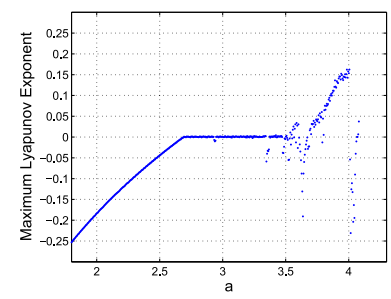

(b)

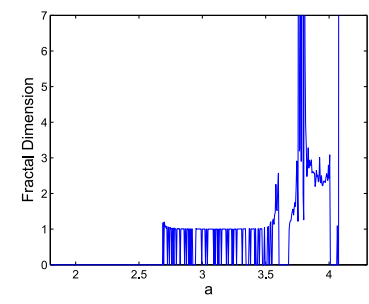

(c)

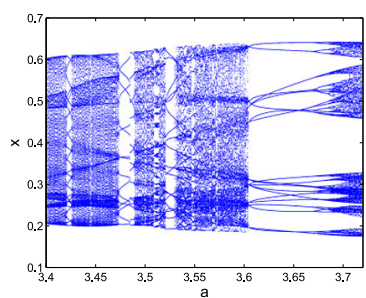

(d)

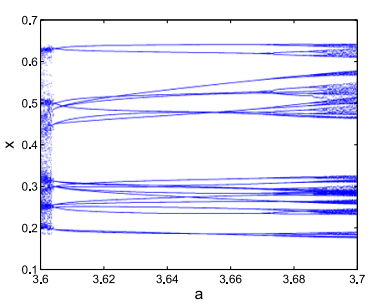

(e)

Figure 6 Bifurcation diagrams, maximum Lyapunov exponent, and fractal dimensions for system (3). (a) Bifurcation diagram in $(a, x)$ plane for $d=3.5$ and $c=0.2$. (b) Maximum Lyapunov exponents corresponding to (a). (c) Fractal dimensions corresponding to (a). (d)-(e) Local amplification corresponding to (a) for $a \in(3.4,3.72)$ and $a \in(3.5,3.7)$. 


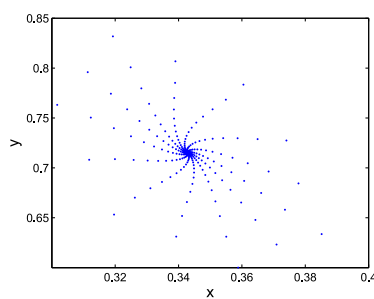

(a) $a=2.61$

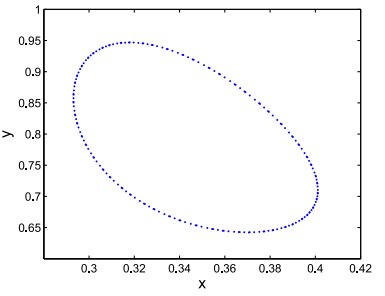

(d) $a=2.74$

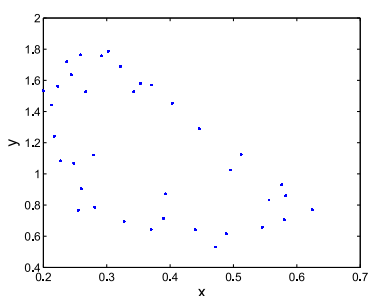

(g) $a=3.51$

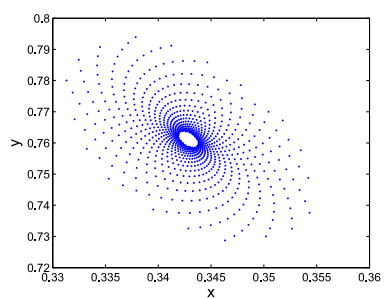

(b) $a=2.68$

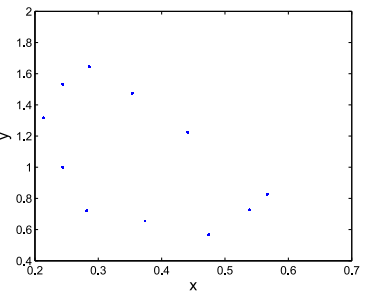

(e) $a=3.35$

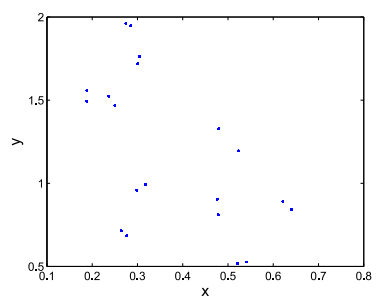

(h) $a=3.65$

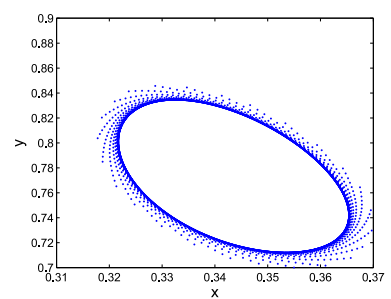

(c) $a=2.7$

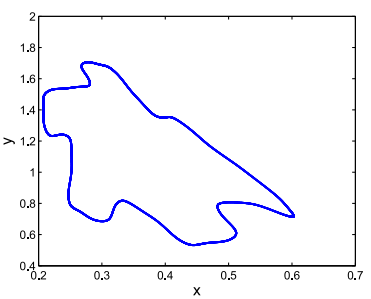

(f) $a=3.4$

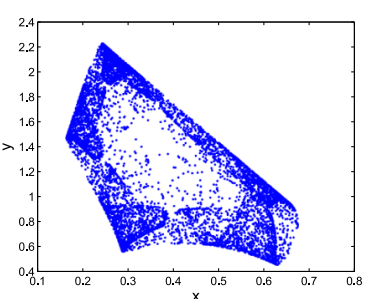

(i) $a=3.85$

Figure 7 Phase portraits for various values of $a$ corresponding to Figure 6(a).

Figure 7, which clearly depicts how a smooth invariant circle bifurcates from the stable fixed point and an invariant circle to chaotic attractors.

\section{Chaos control}

In this section, we apply the state feedback control method [30-32] to stabilize chaotic orbits at an unstable fixed point of system (3).

Consider the following controlled form of system (3):

$$
\left\{\begin{array}{l}
x_{n+1}=a x_{n}\left(1-x_{n}\right)-x_{n} y_{n}+u_{n} \\
y_{n+1}=-c y_{n}+d x_{n} y_{n}
\end{array}\right.
$$

with the following feedback control law as the control force:

$$
u_{n}=-k_{1}\left(x_{n}-x^{*}\right)-k_{2}\left(y_{n}-y^{*}\right)
$$

where $k_{1}$ and $k_{2}$ are the feedback gains, and $\left(x^{*}, y^{*}\right)$ is the positive fixed point of system (3). 
The Jacobian matrix $J$ of the controlled system (17) evaluated at the fixed point $\left(x^{*}, y^{*}\right)$ is given by

$$
J\left(x^{*}, y^{*}\right)=\left(\begin{array}{cc}
-k_{1}+a-2 a x^{*}-y^{*} & -k_{2}-x^{*} \\
d y^{*} & -c+d x^{*}
\end{array}\right),
$$

and the characteristic equation of the Jacobian matrix $J\left(x^{*}, y^{*}\right)$ is

$$
\lambda^{2}-\left(a-c+(d-2 a) x^{*}-y^{*}-k_{1}\right) \lambda+\left(k_{1}-a\right) c+\left(2 a c+a d-d k_{1}-2 a d x^{*}\right) x^{*}+\left(c+d k_{2}\right) y^{*}=0 .
$$

Assume that the eigenvalues are $\lambda_{1}$ and $\lambda_{2}$. Then

$$
\lambda_{1}+\lambda_{2}=a-c+(d-2 a) x^{*}-y^{*}-k_{1}
$$

and

$$
\lambda_{1} \lambda_{2}=a\left(2 x^{*}-1\right)\left(c-d x^{*}\right)+c y^{*}+\left(c-d x^{*}\right) k_{1}+d y^{*} k_{2} .
$$

The lines of marginal stability are determined by the equations $\lambda_{1}= \pm 1$ and $\lambda_{1} \lambda_{2}=1$. These conditions guarantee that the eigenvalues $\lambda_{1}$ and $\lambda_{2}$ have moduli equal to 1 .

Assume that $\lambda_{1} \lambda_{2}=1$. Then from (19) we have

$$
l_{1}: d k_{1}+d(a+a c+d-a d) k_{2}=-(1+c)(a(2+c-d)+d) .
$$

Assume that $\lambda_{1}=1$. Then from (18) and (19) we get

$$
l_{2}: k_{2}=-\frac{1+c}{d} .
$$

Assume that $\lambda_{1}=-1$. Then from (18) and (19) we obtain

$$
l_{3}: 2 d k_{1}+d(a+a c+d-a d) k_{2}=(1-c) d-a(1+c)(3+c-d) .
$$

The stable eigenvalues lie within a triangular region by lines $l_{1}, l_{2}$, and $l_{3}$ (see Figure $8(\mathrm{a})$ ).

Some numerical simulations have been performed to see how the state feedback method controls the unstable fixed point. The parameter values are fixed as $a=3.4, d=3.8$, $c=0.02$. The initial value is $(0.3,1.25)$, and the feedback gains are $k_{1}=0.5$ and $k_{2}=$ -0.04 . Figures $8(\mathrm{~b})$ and $8(\mathrm{c})$ show that a chaotic trajectory is stabilized at the fixed point $(0.315789,1.32632)$.

\section{Conclusions}

In this paper, we have investigated the complex dynamic behaviors of the predator-prey system (3). By using the center manifold theorem and the bifurcation theory we proved that the discrete-time system (3) can undergo a flip bifurcation and a Neimark-Sacker bifurcation. Moreover, system (3) displays much more interesting dynamical behaviors, which include orbits of period- $6,-11,-16,-18,-20,-21,-24,-27$, and -37 , invariant cycles, quasi-periodic orbits, and chaotic sets. They all imply that the predator and prey can coexist at period- $n$ oscillatory balance behaviors or a oscillatory balance behavior, but the 


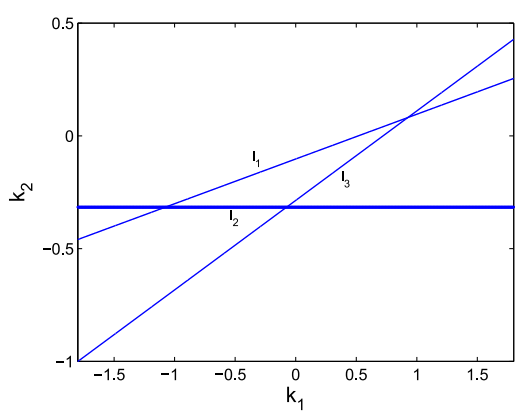

(a)

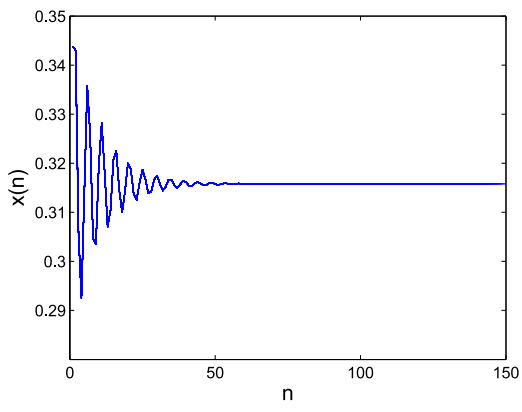

(b)

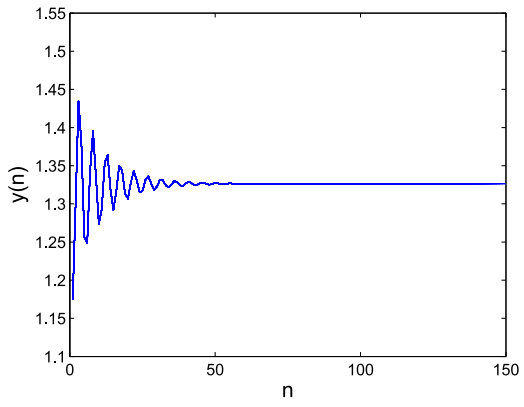

(c)

Figure 8 The region for the stable eigenvalues and the time responses for the states of the controlled system (17). (a) The bounded region for the eigenvalues of the controlled system (17) in the $\left(k_{1}, k_{2}\right)$ plane for $a=3.4, d=3.8$, and $c=0.02$. (b) The time responses for the state $x$ of the controlled system (17) in the $(n, x)$ plane for $a=3.4, d=3.8, c=0.2, k_{1}=0.5, k_{2}=-0.04$. The initial value is $(0.3,1.25)$. (c) The time responses for the state $y$ of the controlled system (17) in the $(n, y)$ plane.

predator-prey system is unstable if a chaotic behavior occurs. In particular, we observe that when the prey is chaotic, the predator will ultimately tend to extinct or tend to a stable fixed point. In comparison with system (1) for $c=0$ in [22], system (3) exhibits different dynamical behaviors in the stability properties and the bifurcation structures. These results show far richer dynamics of the discrete-time model. Finally, we have stabilized the chaotic orbits at an unstable fixed point using the feedback control method.

Competing interests

The authors declare that they have no competing interests.

Authors' contributions

The main idea of this paper was proposed by MZ, ZX, and CL. MZ, ZX, and CL prepared the manuscript initially and performed all the steps of the proofs in this research. All authors read and approved the final manuscript.

\section{Author details}

${ }^{1}$ LMIB - School of Mathematics and Systems Science, Beihang University, Beijing, 100191, China. ${ }^{2}$ Beijing Key Laboratory of Information Service Engineering, Department of General Education, Beijing Union University, Beijing, 100101, China.

\section{Acknowledgements}

The authors would like to thank the reviewers and the editor for very helpful suggestions and comments, which led to improvements of our original paper. ZX is the corresponding author and is supported in part by NNSFC (No. 91420202) and the Project of Construction of Innovative Teams and Teacher Career Development for Universities and Colleges Under Beijing Municipality (CIT and TCD201504041, IDHT20140508). CL is supported by the National Natural Science Foundation of China (Nos. 61134005, 11272024). 


\section{References}

1. Lotka, AJ: Elements of Mathematical Biology. Dover, New York (1956)

2. Volterra, V: Leçons sur la Théorie Mathématique de la Lutte pour la Vie. Gauthier-Villars, Paris (1931)

3. Lindström, T: Qualitative analysis of a predator-prey systems with limit cycles. J. Math. Biol. 31, 541-561 (1993)

4. Ruan, S, Xiao, D: Global analysis in a predator-prey system with nonmonotonic functional response. SIAM J. Appl. Math. 61, 1445-1472 (2001)

5. Cheng, Z, Liu, Y, Cao, J: Dynamical behaviors of a partial-dependent predator-prey system. Chaos Solitons Fractals 28 , 67-75 (2006)

6. Liu, B, Teng, Z, Chen, L: Analysis of a predator-prey model with Holling II functional response concerning impulsive control strategy. J. Comput. Appl. Math. 193, 347-362 (2006)

7. Wang, JL, Wang, KF, Jiang, ZC: Dynamical behaviors of an HTLV-I infection model with intracellular delay and immune activation delay. Adv. Differ. Equ. 2015, 243 (2015)

8. Li, F, Wang, JL: Analysis of an HIV infection model with logistic target-cell growth and cell-to-cell transmission. Chaos Solitons Fractals 81, 136-145 (2015)

9. Murray, JD: Mathematical Biology. Springer, New York (1993)

10. He, ZM, Lai, X: Bifurcations and chaotic behavior of a discrete-time predator-prey system. Nonlinear Anal., Real World Appl. 12, 403-417 (2011)

11. Cheng, LF, Cao, HJ: Bifurcation analysis of a discrete-time ratio-dependent predator-prey model with Allee effect. Commun. Nonlinear Sci. Numer. Simul. 38, 288-302 (2016)

12. He, ZM, Li, B: Complex dynamic behavior of a discrete-time predator-prey system of Holling-III type. Adv. Differ. Equ. 2014, $180(2014)$

13. Choudhury, SR: On bifurcations and chaos in predator-prey models with delay. Chaos Solitons Fractals 2, 393-409 (1992)

14. Fan, M, Agarwal, S: Periodic solutions of nonautonomous discrete predator-prey system of Lotka-Volterra type. Appl. Anal. 81, 801-812 (2002)

15. Jing, ZJ, Yang, J: Bifurcation and chaos in discrete-time predator-prey system. Chaos Solitons Fractals 27, 259-277 (2006)

16. Chen, XW, Fu, XL, Jing, ZJ: Dynamics in a discrete-time predator-prey system with Allee effect. Acta Math. Appl. Sin. 29, 143-164 (2012)

17. Sun, C, Han, M, Lin, Y, Chen, Y: Global qualitative analysis for a predator-prey system with delay. Chaos Solitons Fractals 32, 1582-1596 (2007)

18. Chen, $X W, F u, X L$, Jing, ZJ: Complex dynamics in a discrete-time predator-prey system without Allee effect. Acta Math. Appl. Sin. 29, 355-376 (2013)

19. Liu, X, Xiao, D: Complex dynamic behaviors of a discrete-time predator-prey system. Chaos Solitons Fractals $32,80-94$ (2007)

20. Jiang, XW, Zhan, XS, Guan, ZH, Zhang, XH, Yu, L: Neimark-Sacker bifurcation analysis on a numerical discretization of Gause-type predator-prey model with delay. J. Franklin Inst. 352, 1-15 (2015)

21. Jiang, XW, Ding, L, Guan, ZH, Yuan, FS: Bifurcation and chaotic behavior of a discrete-time Ricardo-Malthus model. Nonlinear Dyn. 71, 437-446 (2013)

22. Chen, $X W$, Fu, XL, Jing, ZJ: Dynamics in a discrete-time predator-prey system with Allee effect. Acta Math. Appl. Sinica (Engl. Ser.) 29, 143-164 (2013). doi:10.1007/s10255-013-0207-5

23. Guckenheimer, J, Holmes, P: Nonlinear Oscillations, Dynamical Systems and Bifurcations of Vector Fields. Springer, New York (1983)

24. Winggins, S: Introduction to Applied Nonlinear Dynamical System and Chaos. Springer, New York (1990)

25. Elaydi, S: Discrete Chaos: With Applications in Science and Engineering, 2nd edn. Chapman \& Hall/CRC, Boca Raton (2008)

26. Alligood, KT, Sauer, TD, Yorke, JA: Chaos: An Introduction to Dynamical Systems, p. 203. Springer, New York (1996)

27. Ott, E: Chaos in Dynamical Systems, 2nd edn., p. 141. Cambridge University Press, Cambridge (2002)

28. Cartwright, JHE: Nonlinear stiffness, Lyapunov exponents, and attractor dimension. Phys. Lett. A 264, $298-302$ (1999)

29. Kaplan, JL, Yorke, JA: A regime observed in a fluid flow model of Lorenz. Commun. Math. Phys. 67, $93-108$ (1979)

30. Chen, G, Dong, X: From Chaos to Order: Perspectives, Methodologies, and Applications. World Scientific, Singapore (1998)

31. Elaydi, S: An Introduction to Difference Equations, 3rd edn. Springer, New York (2005)

32. Lynch, S: Dynamical Systems with Applications Using Mathematica. Birkhäuser, Boston (2007) 